CUBO A Mathematical Journal Vol.13, № 03, (117-139). October 2011

\title{
Pseudo-Almost Automorphic Solutions to Some Second-Order Differential Equations
}

\author{
Toka Diagana \\ Department of Mathematics, \\ Howard University, \\ 2441 6th Street N.W., Washington, D.C. 20059 - USA. \\ email: tdiagana@howard.edu \\ and \\ Ahmed Mohamed \\ Department of Mathematics, \\ Howard University, \\ 2441 6th Street N.W., Washington, D.C. 20059 - USA. \\ email: ahmohamed@howard.edu
}

\begin{abstract}
In this paper we study and obtain the existence of pseudo-almost automorphic solutions to some classes of second-order abstract differential equations on a Hilbert space. To illustrate our abstract results, we discuss the existence of pseudo almost automorphic solutions to the $\mathrm{N}$-dimensional Sine-Gordon boundary value problem.
\end{abstract}

\section{RESUMEN}

En este trabajo se estudia y obtiene la existencia de soluciones casi-seudo automorfas a algunas clases de ecuaciones diferenciales abstractas de segundo orden en un espacio de Hilbert. Para ilustrar nuestros resultados abstractos, se discute la existencia de 
soluciones casi-seudo automorfas en el problema de contorno N-dimensional de SineGordon .

Keywords. exponential stability, sectorial operator, hyperbolic semigroup, almost automorphic; pseudo-almost automorphic; autonomous second-order differential equation; Sine-Gordon equation. amenability, Banach modules, module amenability, weak module amenability, semigroup algebra, inverse semigroup.

Mathematics Subject Classification: 43A60; 34B05; 34C27; 42A75; 47D06; 35L90.

\section{Introduction}

In Leiva 36, the existence of (exponentially stable) bounded solutions and almost periodic solutions to the second-order systems of differential equations given by

$$
u^{\prime \prime}(t)+c u^{\prime}(t)+d A u+k H(u)=P(t), \quad u \in \mathbb{R}^{n}, \quad t \quad \in \mathbb{R}
$$

where $A$ is an $n \times n$-matrix whose eigenvalues are positive, $c, d, k$ are positive constants, $H: \mathbb{R}^{n} \mapsto$ $\mathbb{R}^{n}$ is a locally Lipschitz function, $\mathrm{P}: \mathbb{R} \mapsto \mathbb{R}^{n}$ is a bounded continuous function, were established.

In this paper, using techniques developped in [36], we obtain some reasonable sufficient conditions, which do guarantee the existence of pseudo-almost automorphic solutions to

$$
u^{\prime \prime}(t)+a u^{\prime}(t)+b A u=f(t, u), \quad t \quad \in \mathbb{R},
$$

where $A: D(A) \subset \mathbb{H} \mapsto \mathbb{H}$ is a self-adjoint linear operator whose spectrum consists of isolated eigenvalues $0<\lambda_{1}<\lambda_{2}<\ldots<\lambda_{n} \rightarrow \infty$ with each eigenvalue having a finite multiplicity $\gamma_{j}$ equals to the multiplicity of the corresponding eigenspace, $a, b>$ are constants, and the function $f: \mathbb{R} \times \mathbb{H} \mapsto \mathbb{H}$ is pseudo-almost automorphic function satisfying some additional conditions.

For that, the main idea consists of rewriting Eq. (1.2) as a first-order differential equation on $\mathbb{X}:=\mathbb{H} \times \mathbb{H}$ involving the $2 \times 2$-operator matrix $\mathcal{B}$. Indeed, if $u$ is differential, setting $z:=\left(\begin{array}{c}u \\ u^{\prime}\end{array}\right)$, Eq. (1.2) can be rewritten in the Hilbert space $\mathbb{X}$ in the following form

$$
z^{\prime}(t)=\mathcal{B} z(t)+F(t, z(t)), t \in \mathbb{R}
$$

where $\mathcal{B}$ is the $2 \times 2$-operator matrix defined by

$$
\mathcal{B}=\left(\begin{array}{cc}
0 & \mathrm{I}_{\mathbb{H}} \\
-\mathrm{bA} & -\mathrm{aI}_{\mathbb{H}}
\end{array}\right)
$$


whose domain $\mathrm{D}(\mathcal{B})$ is given by $\mathrm{D}(\mathcal{B})=\mathrm{D}(\mathcal{A}) \times \mathbb{H}$. Moreover, the semilinear term $\mathrm{F}$ appearing in Eq. (1.3) is defined on $\mathbb{R} \times \mathbb{X}_{\alpha}$ for some $\alpha \in(0,1)$ by

$$
F(t, u, v)=\left(\begin{array}{c}
0 \\
f(t, u)
\end{array}\right),
$$

where $\mathbb{X}_{\alpha}$ is an intermediate space (see assumption(H.2)).

Under some reasonable assumptions, it will be shown that the linear operator matrix $\mathcal{B}$ is sectorial and that its associated semigroup is exponentially stable.

The concept of pseudo almost automorphy is a powerful generalization of both the notion of almost automorphy due to Bochner (see [46]) and that of pseudo almost periodicity due to Zhang (see [21]), which has recently been introduced in the literature by Liang et al. 39, 52, 53. Such a concept has recently generated several developments and extensions, see, e.g., [18], 20, 29, 30], and [40].

The existence of almost periodic solutions to second-order differential equations constitutes one of the most important topics in qualitative theory of differential equations due essentially to their applications such thermoelastic plate equations [12, 37] or telegraph equation [43] or SineGordon equations [36. Some contributions on the maximal regularity, bounded, almost periodic, asymptotically almost periodic solutions to abstract second-order differential and partial differential equations have recently been made, among them are [9, [10, [18, [20, 29], 30, 39], [40, [52, [53, 54, 55, and 56. However, to the best of our knowledge, the existence of pseudo-almost automorphic solutions to second-order differential equations of the form Eq. (1.2) is an untreated original question, which in fact is the main motivation of the present paper.

The paper is organized as follows: Section 2 is devoted to preliminaries facts needed in the sequel. In particular, facts related to sectorial operators and hyperbolic semigroups are discussed. In addition, basic definitions and classical results on the concept of pseudo-almost automorphy are also given. In Sections 3 and 4, we prove the main result. In Section 5, we provide the reader with a few examples to illustrate our main result.

\section{Preliminaries}

In the sequel, $A: D(A) \subset \mathbb{H} \mapsto \mathbb{H}$ stands for a self-adjoint (possibly unbounded) linear operator on the Hilbert space $\mathbb{H}$ whose spectrum consists of isolated eigenvalues

$$
0<\lambda_{1}<\lambda_{2}<\ldots<\lambda_{n} \rightarrow \infty
$$

with each eigenvalue having a finite multiplicity $\gamma_{j}$ equals to the multiplicity of the corresponding eigenspace. Let $\left\{\boldsymbol{e}_{j}^{k}\right\}$ be a (complete) orthonormal sequence of eigenvectors associated with the 
eigenvalues $\left\{\lambda_{j}\right\}_{j} \geq 1$. Clearly, for each

$$
\begin{gathered}
u \in D(A):=\left\{u \in \mathbb{H}: \sum_{j=1}^{\infty} \lambda_{j}^{2}\left\|E_{j} u\right\|^{2}<\infty\right\}, \\
A u=\sum_{j=1}^{\infty} \lambda_{j} \sum_{k=1}^{\gamma_{j}}\left\langle u, e_{j}^{k}\right\rangle e_{j}^{k}=\sum_{j=1}^{\infty} \lambda_{j} E_{j} u
\end{gathered}
$$

where $E_{j} u=\sum_{k=1}^{\gamma_{j}}\left\langle u, e_{j}^{k}\right\rangle e_{j}^{k}$.

Note that $\left\{E_{j}\right\}_{j \geq 1}$ is a sequence of orthogonal projections on $\mathbb{H}$. Moreover, each $u \in \mathbb{H}$ can written as follows:

$$
\mathfrak{u}=\sum_{j=1}^{\infty} E_{j} u .
$$

It should also be mentioned that the operator $-A$ is the infinitesimal generator of an analytic semigroup $\{S(t)\}_{t \geq 0}$, which is explicitly expressed in terms of those orthogonal projections $E_{j}$ by, for all $u \in \mathbb{H}$,

$$
S(t) u=\sum_{j=1}^{\infty} e^{-\lambda_{j} t} E_{j} u .
$$

In addition, the fractional powers $A^{r}(r \geq 0)$ of $A$ exist and are given by

$$
D\left(A^{r}\right)=\left\{u \in \mathbb{H}: \sum_{j=1}^{\infty} \lambda_{j}^{2 r}\left\|E_{j} u\right\|^{2}<\infty\right\}
$$

and

$$
A^{r} u=\sum_{j=1}^{\infty} \lambda_{j}^{2 r} E_{j} u, \quad \forall u \in D\left(A^{r}\right)
$$

Let $(\mathbb{X},\|\cdot\|)$ be a Banach space. If $L$ is a linear operator on the Banach space $\mathbb{X}$, then, $D(L)$, $\rho(L), \sigma(L), N(L)$, and $R(L)$, stand respectively for the domain, resolvent, spectrum, null-space or kernel, and range of the operator L. Moreover, one sets $R(\lambda, L):=(\lambda I-L)^{-1}$ for all $\langle\in \rho(A)$. Furthermore, we set $Q=I-P$ for a projection $P$. If $Y, \mathbb{Z}$ are Banach spaces, then the space $B(\mathcal{Y}, \mathbb{Z})$ denotes the collection of all bounded linear operators from $\mathcal{Y}$ into $\mathbb{Z}$ equipped with its natural topology. This is simply denoted by $\mathrm{B}(\mathcal{Y})$ when $\mathcal{Y}=\mathbb{Z}$.

\section{Sectorial Linear Operators}

Definition 3.1. A linear operator $L: D(L) \subset \mathbb{X} \mapsto \mathbb{X}$ (not necessarily densely defined) is said to be sectorial if the following hold: there exist constants $\omega \in \mathbb{R}, \theta \in\left(\frac{\pi}{2}, \pi\right)$, and $M>0$ such that 
$\rho(\mathrm{L}) \supset S_{\theta, \omega}$,

$$
\begin{aligned}
& S_{\theta, \omega}:=\{\lambda \in \mathbb{C}: \lambda \neq \omega, \quad|\arg (\lambda-\omega)|<\theta\}, \\
& \text { and }\|R(\lambda, L)\| \leq \frac{M}{|\lambda-\omega|}, \quad \lambda \in S_{\theta, \omega} .
\end{aligned}
$$

The class of sectorial operators is very rich and contains most of classical operators encountered in the literature. Two examples of sectorial operators are given below.

Example 3.1. Let $p \geq 1$ and let $\mathbb{X}=L^{p}(0,1)$ be the Lebesgue space equipped with its norm $\|\cdot\|_{p}$ defined by

$$
\|\varphi\|_{p}=\left(\int_{0}^{1}|\varphi(x)|^{p} \mathrm{~d} x\right)^{1 / p} .
$$

Define the linear operator $A$ on $L^{p}(0,1)$ by

$$
D(A)=\left\{u \in W^{2, p}(0,1): u^{\prime}(0)=u^{\prime}(1)=0\right\}, \quad A(\varphi)=\varphi^{\prime \prime}, \quad \forall \varphi \in D(A) .
$$

It can be checked that the operator $A$ is sectorial on $L^{p}(0,1)$.

Example 3.2. Let $p \geq 1$ and let $\Omega \subset \mathbb{R}^{\mathrm{d}}$ be open bounded subset with $C^{2}$ boundary $\partial \Omega$. Let $\mathbb{X}:=\mathrm{L}^{\mathfrak{p}}(\Omega)$ be the Lebesgue space equipped with the norm, $\|\cdot\|_{p}$ defined by,

$$
\|\varphi\|_{p}=\left(\int_{\Omega}|\varphi(x)|^{p} \mathrm{dx}\right)^{1 / p}
$$

Define the operator $A$ as follows:

$$
\mathrm{D}(\mathrm{A})=\mathrm{W}^{2, p}(\Omega) \cap W_{0}^{1, p}(\Omega), \quad A(\varphi)=\Delta \varphi, \quad \forall \varphi \in \mathrm{D}(\mathrm{A}),
$$

where $\Delta=\sum_{k=1}^{d} \frac{\partial^{2}}{\partial x_{k}^{2}}$ is the Laplace operator.

It can be checked that the operator $A$ is sectorial on $L^{p}(\Omega)$.

It is well-known that [41] if $A$ is sectorial, then it generates an analytic semigroup $(T(t))_{t \geq 0}$, which maps $(0, \infty)$ into $B(\mathbb{X})$ and such that there exist $M_{0}, M_{1}>0$ with

$$
\begin{aligned}
& \|T(t)\| \leq M_{0} e^{\omega t}, \quad t>0, \\
& \|t(A-\omega) T(t)\| \leq M_{1} e^{\omega t}, \quad t>0 .
\end{aligned}
$$

In this paper, we suppose that the semigroup $(T(t))_{t \geq 0}$ is hyperbolic, that is, there exist a projection $P$ and constants $M, \delta>0$ such that $T(t)$ commutes with $P, N(P)$ is invariant with respect to $T(t), T(t): R(Q) \mapsto R(Q)$ is invertible, and the following hold

$$
\|T(t) P x\| \leq M e^{-\delta t}\|x\| \quad \text { for } t \geq 0,
$$




$$
\|\mathrm{T}(\mathrm{t}) \mathrm{Q} x\| \leq M e^{\delta t}\|x\| \quad \text { for } t \leq 0
$$

where $\mathrm{Q}:=\mathrm{I}-\mathrm{P}$ and, for $\mathrm{t} \leq 0, \mathrm{~T}(\mathrm{t}):=(\mathrm{T}(-\mathrm{t}))^{-1}$.

Recall that the analytic semigroup $(T(t))_{t \geq 0}$ associated with $A$ is hyperbolic if and only if

$$
\sigma(A) \cap i \mathbb{R}=\emptyset
$$

see details in [28, Prop. 1.15, pp.305].

Definition 3.2. Let $\alpha \in(0,1)$. A Banach space $\left(\mathbb{X}_{\alpha},\|\cdot\|_{\alpha}\right)$ is said to be an intermediate space between $\mathrm{D}(\mathrm{A})$ and $\mathbb{X}$, or a space of class $\mathcal{J}_{\alpha}$, if $\mathrm{D}(A) \subset \mathbb{X}_{\alpha} \subset \mathbb{X}$ and there is a constant $\mathrm{c}>0$ such that

$$
\|x\|_{\alpha} \leq \mathrm{c}\|x\|^{1-\alpha}\|x\|_{A}^{\alpha}, \quad x \in \mathrm{D}(A)
$$

where $\|\cdot\|_{A}$ is the graph norm of $A$.

Concrete examples of $\mathbb{X}_{\alpha}$ include $\mathrm{D}\left(\left(-\mathrm{A}^{\alpha}\right)\right)$ for $\alpha \in(0,1)$, the domains of the fractional powers of $A$, the real interpolation spaces $D_{A}(\alpha, \infty), \alpha \in(0,1)$, defined as the space of all $x \in \mathbb{X}$ such

$$
[x]_{\alpha}=\sup _{0<t \leq 1}\left\|t^{1-\alpha} A T(t) x\right\|<\infty
$$

with the norm

$$
\|x\|_{\alpha}=\|x\|+[x]_{\alpha}
$$

the abstract Hölder spaces $D_{A}(\alpha):=\overline{\mathrm{D}(A)}^{\|\cdot\|_{\alpha}}$ as well as the complex interpolation spaces $[\mathbb{X}, \mathrm{D}(A)]_{\alpha}$, see Lunardi 41 for details.

For a hyperbolic analytic semigroup $(T(t))_{t \geq 0}$, one can easily check that similar estimations as both Eq. (3.5) and Eq. (3.6) still hold with the $\alpha$-norms $\|\cdot\|_{\alpha}$. In fact, as the part of $A$ in $\mathrm{R}(\mathrm{Q})$ is bounded, it follows from Eq. (3.6) that

$$
\|A T(t) Q x\| \leq C^{\prime} e^{\delta t}\|x\| \text { fort } \leq 0
$$

Hence, from Eq. (3.7) there exists a constant $c(\alpha)>0$ such that

$$
\|\mathrm{T}(\mathrm{t}) \mathrm{Q} x\|_{\alpha} \leq \mathrm{c}(\alpha) e^{\delta \mathrm{t}}\|\mathrm{x}\| \quad \text { for } \mathrm{t} \leq 0
$$

In addition to the above, the following holds

$$
\|\mathrm{T}(\mathrm{t}) \mathrm{Px}\|_{\alpha} \leq\|\mathrm{T}(1)\|_{\mathrm{B}\left(\mathbb{X}, \mathbb{X}_{\alpha}\right)}\|\mathrm{T}(\mathrm{t}-1) \mathrm{P} x\|, \quad \mathrm{t} \geq 1
$$

and hence from Eq. (3.5), one obtains

$$
\|\mathrm{T}(\mathrm{t}) \mathrm{Px}\|_{\alpha} \leq \mathrm{M}^{\prime} \mathrm{e}^{-\delta \mathrm{t}}\|x\|, \quad \mathrm{t} \geq 1,
$$


where $M^{\prime}$ depends on $\alpha$. For $t \in(0,1]$, by Eq. (3.4) and Eq. (3.7),

$$
\|\mathrm{T}(\mathrm{t}) \mathrm{Px}\|_{\alpha} \leq \mathrm{M}^{\prime \prime} \mathrm{t}^{-\alpha}\|\mathrm{x}\| .
$$

Hence, there exist constants $M(\alpha)>0$ and $\gamma>0$ such that

$$
\|\mathrm{T}(\mathrm{t}) \mathrm{Px}\|_{\alpha} \leq M(\alpha) \mathrm{t}^{-\alpha} e^{-\gamma \mathrm{t}}\|x\| \quad \text { for } \mathrm{t}>0 .
$$

\subsection{Pseudo-Almost Automorphic Functions}

Let $\mathrm{BC}(\mathbb{R}, \mathbb{X})$ (respectively,

$\mathrm{BC}(\mathbb{R} \times \mathcal{Y}, \mathbb{X}))$ denote the collection of all $\mathbb{X}$-valued bounded continuous functions (respectively, the class of jointly bounded continuous functions $\mathrm{F}: \mathbb{R} \times \mathcal{Y} \mapsto \mathbb{X})$. The space $\mathrm{BC}(\mathbb{R}, \mathbb{X})$ equipped with the sup norm defined by

$$
\|\mathfrak{u}\|_{\infty}=\sup _{\mathfrak{t} \in \mathbb{R}}\|\mathfrak{u}(\mathrm{t})\|
$$

is a Banach space. Furthermore, $\mathrm{C}(\mathbb{R}, \mathcal{Y})$ (respectively, $\mathrm{C}(\mathbb{R} \times \mathcal{Y}, \mathbb{X})$ ) denotes the class of continuous functions from $\mathbb{R}$ into $\mathcal{Y}$ (respectively, the class of jointly continuous functions $\mathrm{F}: \mathbb{R} \times \mathcal{Y} \mapsto \mathbb{X}$ ).

Definition 3.3. A function $\mathrm{f} \in \mathrm{C}(\mathbb{R}, \mathbb{X})$ is said to be almost automorphic if for every sequence of real numbers $\left(s_{\mathfrak{n}}^{\prime}\right)_{n \in \mathbb{N}}$, there exists a subsequence $\left(s_{\mathfrak{n}}\right)_{\mathfrak{n} \in \mathbb{N}}$ such that

$$
g(t):=\lim _{n \rightarrow \infty} f\left(t+s_{n}\right)
$$

is well defined for each $\mathrm{t} \in \mathbb{R}$, and

$$
\lim _{n \rightarrow \infty} g\left(t-s_{n}\right)=f(t)
$$

for each $\mathrm{t} \in \mathbb{R}$.

If the convergence above is uniform in $t \in \mathbb{R}$, then $\mathrm{f}$ is almost periodic in the classical Bochner's sense. Denote by $A A(\mathbb{X})$ the collection of all almost automorphic functions $\mathbb{R} \mapsto \mathbb{X}$. Note that $A A(\mathbb{X})$ equipped with the sup-norm turns out to be a Banach space.

Among other things, almost automorphic functions satisfy the following properties.

Theorem 3.1. $\mid 46]$ If $f, f_{1}, f_{2} \in A A(\mathbb{X})$, then

(i) $f_{1}+f_{2} \in A A(\mathbb{X})$,

(ii) $\lambda f \in A A(\mathbb{X})$ for any scalar $\lambda$,

(iii) $\mathrm{f}_{\alpha} \in \mathrm{AA}(\mathbb{X})$ where $\mathrm{f}_{\alpha}: \mathbb{R} \rightarrow \mathbb{X}$ is defined by $\mathrm{f}_{\alpha}(\cdot)=\mathrm{f}(\cdot+\alpha)$,

(iv) the range $\mathcal{R}_{\mathrm{f}}:=\{\mathrm{f}(\mathrm{t}): \mathrm{t} \in \mathbb{R}\}$ is relatively compact in $\mathbb{X}$, thus $\mathrm{f}$ is bounded in norm,

(v) if $\mathrm{f}_{n} \rightarrow \mathrm{f}$ uniformly on $\mathbb{R}$ where each $\mathrm{f}_{\mathrm{n}} \in \mathrm{AA}(\mathbb{X})$, then $\mathrm{f} \in \mathrm{AA}(\mathbb{X})$ too. 
In addition to the above-mentioned properties, we have the the following property due to Bugajewski and Diagana [15]:

(vi) if $g \in L^{1}(\mathbb{R})$, then $f * g \in A A(\mathbb{R})$, where $f * g$ is the convolution of $f$ with $g$ on $\mathbb{R}$.

Let $\left(\mathcal{Y},\|\cdot\|_{\mathcal{Y}}\right)$ be another Banach space.

Definition 3.4. A jointly continuous function $\mathrm{F}: \mathbb{R} \times \mathcal{Y} \mapsto \mathbb{X}$ is said to be almost automorphic in $\mathrm{t} \in \mathbb{R}$ if $\mathrm{t} \mapsto \mathrm{F}(\mathrm{t}, \mathrm{x})$ is almost automorphic for all $\mathrm{x} \in \mathrm{K}(\mathrm{K} \subset \mathcal{Y}$ being any bounded subset). Equivalently, for every sequence of real numbers $\left(s_{n}^{\prime}\right)_{n \in \mathbb{N}}$, there exists a subsequence $\left(s_{n}\right)_{n \in \mathbb{N}}$ such that

$$
G(t, x):=\lim _{n \rightarrow \infty} F\left(t+s_{n}, x\right)
$$

is well defined in $\mathrm{t} \in \mathbb{R}$ and for each $\mathrm{x} \in \mathrm{K}$, and

$$
\lim _{n \rightarrow \infty} G\left(t-s_{n}, x\right)=F(t, x)
$$

for all $\mathrm{t} \in \mathbb{R}$ and $\mathrm{x} \in \mathrm{K}$.

The collection of such functions will be denoted by $A A(\mathcal{Y}, \mathbb{X})$.

For more on almost automorphic functions and related issues, we refer the reader to the excellent book by N'Guérékata [46].

Define

$$
\operatorname{PAP}_{\mathrm{O}}(\mathbb{R}, \mathbb{X}):=\left\{f \in B C(\mathbb{R}, \mathbb{X}): \lim _{\mathrm{T} \rightarrow \infty} \frac{1}{2 \mathrm{~T}} \int_{-\mathrm{T}}^{\mathrm{T}}\|f(s)\| \mathrm{d} s=0\right\} .
$$

Similarly, $\operatorname{PAP}_{0}(\mathcal{Y}, \mathbb{X})$ will denote the collection of all bounded continuous functions $\mathrm{F}: \mathbb{R} \times$ $\mathcal{Y} \mapsto \mathbb{X}$ such that

$$
\lim _{T \rightarrow \infty} \frac{1}{2 T} \int_{-T}^{T}\|F(s, x)\| d s=0
$$

uniformly in $\mathrm{x} \in \mathrm{K}$, where $\mathrm{K} \subset \mathcal{Y}$ is any bounded subset.

Definition 3.5. (Liang et al. [39] and Xiao et al. [52]) A function $\mathrm{f} \in \mathrm{BC}(\mathbb{R}, \mathbb{X})$ is called pseudo almost automorphic if it can be expressed as $\mathrm{f}=\mathrm{g}+\phi$, where $\mathrm{g} \in \mathrm{AA}(\mathbb{X})$ and $\phi \in \mathrm{PAP}_{0}(\mathbb{X})$. The collection of such functions will be denoted by $\operatorname{PAA}(\mathbb{X})$.

The functions $\mathrm{g}$ and $\phi$ appearing in Definition 3.5 are respectively called the almost automorphic and the ergodic perturbation components of $\mathrm{f}$.

Definition 3.6. A bounded continuous function $\mathrm{F}: \mathbb{R} \times \mathcal{Y} \mapsto \mathbb{X}$ belongs to $\mathrm{AA}(\mathrm{Y}, \mathbb{X})$ whenever it can be expressed as $\mathrm{F}=\mathrm{G}+\Phi$, where $\mathrm{G} \in \mathrm{AA}(\mathcal{Y}, \mathbb{X})$ and $\Phi \in \operatorname{PAP}_{0}(\mathcal{Y}, \mathbb{X})$. The collection of such functions will be denoted by $\operatorname{PAA}(\mathcal{Y}, \mathbb{X})$.

We now collect a few useful properties of pseudo almost automorphic functions. 
Proposition 3.1. If $g \in L^{1}(\mathbb{R}), f \in P A A(\mathbb{R})$, then $f * g \in P A A(\mathbb{R})$, where $f * g$ is the convolution of $f$ with $g$ on $\mathbb{R}$.

The proof of Proposition 3.1 is based upon [15] and [16.

A substantial result is the next theorem, which is due to Xiao et al. [52.

Theorem 3.2. [52] The space $\operatorname{PAA}(\mathbb{X})$ equipped with the sup norm $\|\cdot\|_{\infty}$ is a Banach space.

The next composition result, that is Theorem 3.3, is a consequence of [40, Theorem 2.4] and is crucial for the proof of the main result of the paper.

Theorem 3.3. Suppose $\mathrm{f}: \mathbb{R} \times \mathcal{Y} \mapsto \mathbb{X}$ belongs to $\operatorname{PAA}(\mathcal{Y}, \mathbb{X}) ; \mathrm{f}=\mathrm{g}+\mathrm{h}$, with $\mathrm{x} \mapsto \mathrm{g}(\mathrm{t}, \mathrm{x})$ being uniformly continuous on each bounded subset $\mathrm{K}$ of $\mathcal{Y}$ uniformly in $\mathrm{t} \in \mathbb{R}$, that is, for each $\varepsilon>0$ there exists $\delta>0$ such that $\mathrm{x}, \mathrm{y} \in \mathrm{K}$ and $\|\mathrm{x}-\mathrm{y}\|<\delta$ yields $\|\mathrm{g}(\mathrm{t}, \mathrm{x})-\mathrm{g}(\mathrm{t}, \mathrm{y})\|<\varepsilon$ for all $\mathrm{t} \in \mathbb{R}$. Furthermore, we suppose that there exists $\mathrm{L}>0$ such that

$$
\|f(t, x)-f(t, y)\| \leq L\|x-y\|_{\mathcal{Y}}
$$

for all $\mathrm{x}, \mathrm{y} \in \mathcal{Y}$ and $\mathrm{t} \in \mathbb{R}$.

Then the function defined by $\mathrm{h}(\mathrm{t})=\mathrm{f}(\mathrm{t}, \varphi(\mathrm{t}))$ belongs to $\operatorname{PAA}(\mathbb{X})$ provided $\varphi \in \operatorname{PAA}(\mathcal{Y})$.

We also have:

Theorem 3.4. 52] If $\mathrm{f}: \mathbb{R} \times \mathcal{Y} \mapsto \mathbb{X}$ belongs to $\operatorname{PAA}(\mathcal{Y}, \mathbb{X})$ and if $\mathrm{x} \mapsto \mathrm{f}(\mathrm{t}, \mathrm{x})$ is uniformly continuous on each bounded subset $\mathrm{K}$ of $\mathcal{Y}$ uniformly in $\mathrm{t} \in \mathbb{R}$, then the function defined by $\mathrm{h}(\mathrm{t})=$ $\mathrm{f}(\mathrm{t}, \varphi(\mathrm{t}))$ belongs to $\operatorname{PAA}(\mathbb{X})$ provided $\varphi \in \operatorname{PAA}(\mathcal{Y})$.

\section{Main results}

Consider the differential equation

$$
\mathfrak{u}^{\prime}(\mathrm{t})=\mathrm{Lu}(\mathrm{t})+\mathrm{F}(\mathrm{t}, \mathrm{u}(\mathrm{t})), \quad \mathrm{t} \in \mathbb{R},
$$

where $\mathrm{L}: \mathrm{D}(\mathrm{L}) \subset \mathbb{X} \mapsto \mathbb{X}$ is sectorial and $\mathrm{F}: \mathbb{R} \times \mathbb{X}_{\alpha} \mapsto \mathbb{X}$ is jointly continuous.

Fix once and for all $\alpha, \beta$ such that $0 \leq \alpha<\beta<1$. To study the existence and uniqueness of pseudo-almost automorphic solutions to Eq. (4.1) we make the following additional assumptions

(H.1) The operator $L$ is sectorial on $\mathbb{X}$ and generates a hyperbolic (analytic) semigroup $(T(t))_{t \geq 0}$.

(H.2) Let $0<\alpha<1$. Then $\mathbb{X}_{\alpha}=\mathrm{D}\left(\left(-\mathrm{A}^{\alpha}\right)\right)$, or $\mathbb{X}_{\alpha}=\mathrm{D}_{\mathcal{A}}(\alpha, p), 1 \leq p \leq+\infty$, or $\mathbb{X}_{\alpha}=\mathrm{D}_{\mathcal{A}}(\alpha)$, or $\mathbb{X}_{\alpha}=[\mathbb{X}, D(A)]_{\alpha}$. 
(H.3) The function $F: \mathbb{R} \times \mathbb{X} \mapsto \mathbb{X}$ is given such that $u \mapsto F(t, u)$ is unformly continuous on each bounded subset $B$ of $\mathbb{X}$ uniformly in $t \in \mathbb{R}$. Furthermore, $F$ is Lipschitz in the following sense: there exists $L>0$ for which

$$
\|\mathrm{F}(\mathrm{t}, \mathrm{u})-\mathrm{F}(\mathrm{t}, \mathrm{v})\|_{\beta} \leq \mathrm{L}\|\mathrm{u}-\mathrm{v}\|_{\alpha}
$$

for all $u, v \in \mathbb{X}$ and $t \in \mathbb{R}$.

Set $S_{1} u(t):=S_{11} u(t)-S_{12} u(t)$ and $S_{2} u=S_{22} u-S_{23} u$, where

$$
S_{11} u(t):=\int_{-\infty}^{t} T(t-s) P F_{1}(s, u(s)) d s, \quad S_{12} u(t):=\int_{t}^{\infty} T(t-s) Q F_{1}(s, u(s)) d s
$$

for all $t \in \mathbb{R}$.

Definition 4.1. Under assumption (H.1), a function $\mathrm{u}: \mathbb{R} \mapsto \mathbb{X}_{\alpha}$ is said to be a mild solution to Eq. (4.1) provided that

$$
u(t)=T(t-s) u(s)+\int_{s}^{t} T(t-r) F(r, u(r)) d r
$$

for each $\forall \mathrm{t} \geq \mathrm{s}, \mathrm{t}, \mathrm{s} \in \mathbb{R}$.

Consider the differential equation

$$
u^{\prime}(t)=L u(t)+g(t), \quad t \in \mathbb{R}
$$

where $\mathrm{g}: \mathbb{R} \mapsto \mathbb{X}$ is continuous.

Theorem 4.1. Under assumptions (H.1)-(H.2), if $\mathrm{g} \in \mathrm{B}(\mathbb{R}, \mathbb{X})$, then we have:

(i) Eq. (4.3) has a unique bounded mild solution $\mathrm{u}: \mathbb{R} \mapsto \mathbb{X}_{\alpha}$, which can be explicitly given by

$$
u(t)=\int_{-\infty}^{t} T(t-s) \operatorname{Pg}(s) d s-\int_{t}^{\infty} T(t-s) \operatorname{Qg}(s) d s
$$

(ii) If $\mathrm{g} \in \operatorname{PAA}\left(\mathbb{X}_{\alpha}\right)$, then $\mathrm{u} \in \operatorname{PAA}\left(\mathbb{X}_{\alpha}\right)$.

Proof. (i) Since $g$ is bounded, we can easily show that $u$ given above is well-defined. Moreover, $u$ satisfies

$$
u(t)=T(t-s) u(s)+\int_{s}^{t} T(t-r) g(r) d r
$$

for each $\forall t \geq s, t, s \in \mathbb{R}$. 
The continuity and uniqueness of $u$ is also clear. For the boundedness in $\mathbb{X}_{\alpha}$, using (3.8) and (3.9), we obtain

$$
\begin{aligned}
\|\mathrm{u}(\mathrm{t})\|_{\alpha} & \leq \mathrm{c}\|\mathrm{u}(\mathrm{t})\|_{\beta} \\
& \leq \mathrm{c} \int_{-\infty}^{\mathrm{t}}\|\mathrm{T}(\mathrm{t}-\mathrm{s}) \operatorname{Pg}(\mathrm{s})\|_{\beta} \mathrm{ds}+\mathrm{c} \int_{\mathrm{t}}^{+\infty}\|\mathrm{T}(\mathrm{t}-\mathrm{s}) \mathrm{Qg}(\mathrm{s})\|_{\beta} \mathrm{d} s \\
& \leq \mathrm{cM}(\beta) \int_{-\infty}^{\mathrm{t}} \mathrm{e}^{-\frac{\delta}{2}(\mathrm{t}-\mathrm{s})}(\mathrm{t}-\mathrm{s})^{-\beta}\|\mathrm{g}(\mathrm{s})\| \mathrm{d} s+\mathrm{cc}(\beta) \int_{\mathrm{t}}^{+\infty} e^{-\delta(s-\mathrm{t})}\|\mathrm{g}(\mathrm{s})\| \mathrm{d} s \\
& \leq \operatorname{cM}(\beta)\|\mathrm{g}\|_{\infty} \int_{0}^{+\infty} e^{-\sigma}\left(\frac{2 \sigma}{\delta}\right)^{-\beta} \frac{2 \mathrm{~d} \sigma}{\delta}+\mathrm{cc}(\beta)\left\|_{\mathrm{g}}\right\|_{\infty} \int_{0}^{+\infty} e^{-\delta \sigma} \mathrm{d} \sigma \\
& \leq \operatorname{cM}(\beta) \delta^{\alpha} \Gamma(1-\beta)\|\mathrm{g}\|_{\infty}+\operatorname{cc}(\beta) \delta^{-1}\|\mathrm{~g}\|_{\infty},
\end{aligned}
$$

and hence

$$
\|x(t)\|_{\alpha} \leq c\|x(t)\|_{\beta} \leq c^{\prime} c\left[M(\beta) \delta^{\beta} \Gamma(1-\beta)+c(\beta) \delta^{-1}\right]\|g\|_{\infty, \alpha}
$$

It remains to prove (ii). For that, we first consider the first integral in the expression of Eq. (4.4) and denote it Su. Now write $g=\phi+\zeta$ where $\phi \in A A\left(\mathbb{X}_{\alpha}\right)$ and $\zeta \in \mathrm{PAP}_{0}\left(\mathbb{X}_{\alpha}\right)$. Clearly, Su can be rewritten as

$$
\left.(S u)(t)=\int_{-\infty}^{t} T(t-s) P \phi(s) d s+\int_{-\infty}^{t} T t-s\right) P \zeta(s) d s .
$$

Set

$$
\Phi(t)=\int_{-\infty}^{t} T(t-s) P \phi(s) d s, \text { and } \Psi(t)=\int_{-\infty}^{t} T(t-s) P \zeta(s) d s
$$

for each $t \in \mathbb{R}$.

The next step consists of showing that $\Phi \in A A\left(\mathbb{X}_{\alpha}\right)$ and $\Psi \in \operatorname{PAP}_{0}\left(\mathbb{X}_{\alpha}\right)$. Indeed, since $\phi \in A A\left(\mathbb{X}_{\alpha}\right)$, for every sequence of real numbers $\left(\tau_{n}^{\prime}\right)_{n \in \mathbb{N}}$ there exists a subsequence $\left(\tau_{n}\right)_{n \in \mathbb{N}}$ such that

$$
\psi(t):=\lim _{n \rightarrow \infty} \phi\left(t+\tau_{n}\right)
$$

is well defined for each $t \in \mathbb{R}$, and

$$
\lim _{n \rightarrow \infty} \psi\left(t-\tau_{n}\right)=\phi(t)
$$

for each $t \in \mathbb{R}$.

Set $\Phi_{1}(t)=\int_{-\infty}^{t} T(t-s) P \psi(s) d s$ for all $t \in \mathbb{R}$.

Now 


$$
\begin{aligned}
\Phi\left(t+\tau_{n}\right)-\Phi_{1}(t) & =\int_{-\infty}^{t+\tau_{n}} T\left(t+\tau_{n}-s\right) P \phi(s) d s-\int_{-\infty}^{t} T(t-s) P \psi(s) d s \\
& =\int_{-\infty}^{t} T(t-s) P \phi\left(s+\tau_{n}\right) d s-\int_{-\infty}^{t} T(t-s) P \psi d s \\
& =\int_{-\infty}^{t} T(t-s) P\left(\phi\left(s+\tau_{n}\right)-\psi(s)\right) d s
\end{aligned}
$$

Using Lebesgue Dominated Convergence Theorem, one can easily see that

$$
\left\|\int_{-\infty}^{t} \mathrm{~T}(\mathrm{t}-\mathrm{s}) \mathrm{P}\left(\phi\left(s+\tau_{n}\right)-\psi(s)\right) d s\right\|_{\alpha} \rightarrow 0 \text { as } \mathrm{n} \rightarrow \infty, \mathrm{t} \in \mathbb{R} .
$$

Thus

$$
\Phi_{1}(t)=\lim _{n \rightarrow \infty} \Phi\left(t+\tau_{n}\right), \quad t \in \mathbb{R} .
$$

Similarly, one can easily see that

$$
\Phi(t)=\lim _{n \rightarrow \infty} \Phi_{1}\left(t-\tau_{n}\right), \quad t \in \mathbb{R} .
$$

Therefore, $\Phi \in A A\left(\mathbb{X}_{\alpha}\right)$.

Let us now show that $\Psi \in \operatorname{PAP}_{0}\left(\mathbb{X}_{\alpha}\right)$. First, note that $s \mapsto \Psi(s)$ is a bounded continuous function. It remains to show that

$$
\lim _{T \rightarrow \infty} \frac{1}{2 T} \int_{-T}^{T}\|\Psi(t)\|_{\alpha} d t=0 .
$$

Again using Eq. (3.9) it follows that

$$
\begin{aligned}
\lim _{T \rightarrow \infty} \frac{1}{2 T} \int_{-T}^{T}\|\Psi(t)\|_{\alpha} d t & \leq \lim _{T \rightarrow \infty} \frac{M(\alpha)}{2 T} \int_{-T}^{T} \int_{0}^{+\infty} s^{-\alpha} e^{-\frac{\delta}{2} s}\|\zeta(t-s)\|_{\alpha} d s d t \\
& \leq \lim _{T \rightarrow \infty} M(\alpha) \int_{0}^{+\infty} s^{-\alpha} e^{-\frac{\delta}{2} s} \frac{1}{2 T} \int_{-T}^{T}\|\zeta(t-s)\|_{\alpha} d t d s .
\end{aligned}
$$

Let

$$
\Gamma_{s}(T)=\frac{1}{2 T} \int_{-T}^{T}\|\zeta(t-s)\|_{\alpha} d t .
$$

Since $\mathrm{PAP}_{0}\left(\mathbb{X}_{\alpha}\right)$ is translation invariant it follows that $t \mapsto \zeta(t-s)$ belongs to $\mathrm{PAP}_{0}\left(\mathbb{X}_{\alpha}\right)$ for each $s \in \mathbb{R}$, and hence

$$
\lim _{T \mapsto \infty} \frac{1}{2 T} \int_{-T}^{T}\|\zeta(t-s)\|_{\alpha} d t=0
$$

for each $s \in \mathbb{R}$. 
One completes the proof by using the Lebesgue Dominated Convergence Theorem and the fact $\Gamma_{\mathrm{s}}(\mathrm{T}) \mapsto 0$ as $\mathrm{T} \rightarrow \infty$ for each $s \in \mathbb{R}$.

The proof for the second integral in the expression of Eq. (4.4) is similar to that of $\mathrm{Su}(\cdot)$ and hence moitted. (In that case, one makes use of Eq. (3.8) rather than Eq. (3.9).)

Using the composition of pseudo-almost automorphic functions and Theorem 4.1, it is easy to see that the following technical lemmas hold.

Lemma 4.1. Under assumptions (H.1)-(H.2)-(H.3), then the integral operator $\mathrm{S}_{1}$ defined above maps $\operatorname{PAA}\left(\mathbb{X}_{\alpha}\right)$ into itself.

Lemma 4.2. Under assumptions (H.1)-(H.2)-(H.3), the integral operator $\mathrm{S}_{1}$ defined above is a contraction whenever $\mathrm{L}$ is small enough.

Proof. Let $v, w \in \operatorname{PAA}\left(\mathbb{X}_{\alpha}\right)$. Now,

$$
\begin{aligned}
\left\|S_{11} v(t)-S_{11} w(t)\right\|_{\alpha} & \leq \int_{-\infty}^{t} M(\alpha)(t-s)^{-\alpha} e^{-\frac{\delta}{2}(t-s)}\left\|F_{1}(s, v(s))-F_{1}(s, w(s))\right\| d s \\
& \leq c \int_{-\infty}^{t} M(\alpha)(t-s)^{-\alpha} e^{-\frac{\delta}{2}(t-s)}\left\|F_{1}(s, v(s))-F_{1}(s, w(s))\right\|_{\beta} d s \\
& \leq \operatorname{LcM}(\alpha) \int_{-\infty}^{t}(t-s)^{-\alpha} e^{-\frac{\delta}{2}(t-s)}\|v(s)-w(s)\| d s \\
& \leq \operatorname{Lc} c M(\alpha) \int_{-\infty}^{t}(t-s)^{-\alpha} e^{-\frac{\delta}{2}(t-s)}\|v(s)-w(s)\|_{\alpha} d s .
\end{aligned}
$$

Similarly,

$$
\begin{aligned}
\left\|S_{12} v(t)-S_{12} w(t)\right\|_{\alpha} & \leq \int_{t}^{\infty} c(\beta) e^{-\delta(t-s)}\left\|F_{1}(s, v(s))-F_{1}(s, w(s))\right\|_{d s} \\
& \leq \operatorname{cc}(\beta) \int_{t}^{\infty} e^{-\delta(t-s)}\left\|F_{1}(s, v(s))-F_{1}(s, w(s))\right\|_{\beta} \mathrm{d} s \\
& \leq \operatorname{Lcc}(\beta) \int_{t}^{\infty} e^{-\delta(t-s)}\|v(s)-w(s)\| d s \\
& \leq \operatorname{Lcc} c(\beta) \int_{t}^{\infty} e^{-\delta(t-s)}\|v(s)-w(s)\|_{\alpha} d s .
\end{aligned}
$$

Consequently,

$$
\left\|S_{1} v-S_{1} w\right\|_{\infty, \alpha} \leq \operatorname{Lcc}^{\prime}\left(M(\alpha) \Gamma(1-\alpha)\left(2 \delta^{-1}\right)^{1-\alpha}+c(\beta) \delta^{-1}\right)\|v-w\|_{\infty, \alpha}
$$

and hence $S_{1}$ is a contraction whenever $L$ is small enough. 
Theorem 4.2. Suppose assumptions (H.1)-(H.2)-(H.3) and that L is small enough, then the autonmous differential equation Eq. (4.1) has a unique pseudo almost automorphic solution u satisfying $\mathrm{u}=\mathrm{S}_{1} \mathrm{u}$.

Proof. This is an immediate consequence of Lemma 4.1. Lemma 4, Lemma 4.2, and the Banach fixed point theorem.

\section{Pseudo Almost Automorphic Solutions to Some Second- Order Differential Equations}

We have previously seen that each $u \in \mathbb{H}$ can be written in terms of the sequence of orthogonal projections $E_{n}$ as follows:

$$
u=\sum_{n=1}^{\infty} \sum_{k=1}^{\gamma_{n}}\left\langle u, e_{n}^{k}\right\rangle e_{n}^{k}=\sum_{n=1}^{\infty} E_{n} u
$$

Moreover, for each $u \in D(A)$,

$$
A u=\sum_{j=1}^{\infty} \lambda_{j} \sum_{k=1}^{\gamma_{j}}\left\langle u, e_{j}^{k}\right\rangle e_{j}^{k}=\sum_{j=1}^{\infty} \lambda_{j} E_{j} u
$$

Therefore, for all $z:=\left(\begin{array}{l}u \\ v\end{array}\right) \in \mathrm{D}=\mathrm{D}(\mathcal{B})=\mathrm{D}(\mathrm{A}) \times \mathbb{H}$, we obtain the following

$$
\begin{aligned}
\mathcal{B} z & \left.=\left(\begin{array}{cc}
0 & \mathrm{I}_{\mathbb{H}} \\
-\mathrm{bA} & -\mathrm{aI}_{\mathbb{H}}
\end{array}\right)\left(\begin{array}{l}
u \\
v
\end{array}\right)=\left(\begin{array}{c}
v \\
\sum_{n=1}^{\infty} E_{n} v \\
-b A u-a v
\end{array}\right) \sum_{n=1}^{\infty} \lambda_{n} E_{n} u-a \sum_{n=1}^{\infty} E_{n} v\right) \\
& =\left(\begin{array}{cc}
0 & 1 \\
-b & 0 \\
E_{n=1} & 0 \\
-b \lambda_{n} & -a
\end{array}\right)\left(\begin{array}{cc}
u \\
0 & E_{n}
\end{array}\right)\left(\begin{array}{cc}
v
\end{array}\right) \\
& =\sum_{n=1}^{\infty} A_{n} P_{n} z,
\end{aligned}
$$


where

$$
P_{n}:=\left(\begin{array}{cc}
E_{n} & 0 \\
0 & E_{n}
\end{array}\right), n \geq 1
$$

and

$$
A_{n}:=\left(\begin{array}{cc}
0 & 1 \\
-b \lambda_{n} & -a
\end{array}\right), n \geq 1 .
$$

Now, the characteristic equation for $A_{n}$ is given by

$$
\lambda^{2}+a \lambda+\lambda_{n} b=0
$$

whose eigenvalues are given by

$$
\lambda_{1}^{n}:=\frac{-a+\sqrt{a^{2}-4 \lambda_{n} b}}{2} \text { and } \lambda_{2}^{n}:=\frac{-a-\sqrt{a^{2}-4 \lambda_{n} b}}{2} .
$$

Since $a>0$ it follows that all roots of Eq. (5.2) are nonzero. Moreover, the real part of each of its roots is: $-a / 2<0$. Therefore, there exists $\omega \in\left(\frac{\pi}{2}, \pi\right)$ such that $\rho(L) \supset S_{\omega}$, where

$$
\mathrm{S}_{\omega}:=\{z \in \mathbb{C} \backslash\{0\}:|\arg z|<\omega\} .
$$

On the other hand, one can show without difficulty that $A_{n}=K_{n}^{-1} J_{n} K_{n}$, where $J_{n}, K_{n}$ and $\mathrm{K}_{n}^{-1}$ are respectively given by

$$
J_{n}=\left(\begin{array}{cc}
\lambda_{1}^{n} & 0 \\
0 & \lambda_{2}^{n}
\end{array}\right), \quad K_{n}=\left(\begin{array}{cc}
1 & 1 \\
\lambda_{1}^{n}(t) & \lambda_{2}^{n}
\end{array}\right) \text {, }
$$

and

$$
K_{n}^{-1}=\frac{1}{\lambda_{1}^{n}-\lambda_{2}^{n}}\left(\begin{array}{cc}
-\lambda_{2}^{n} & 1 \\
\lambda_{1}^{n} & -1
\end{array}\right)
$$

For $\lambda \in S_{\omega}$ and $z \in \mathbb{X}$, one has

$$
\begin{aligned}
R(\lambda, \mathcal{B}) z & =\sum_{n=1}^{\infty}\left(\lambda-A_{n}\right)^{-1} P_{n} z \\
& =\sum_{n=1}^{\infty} K_{n}\left(\lambda-J_{n} P_{n}\right)^{-1} K_{n}^{-1} P_{n} z .
\end{aligned}
$$


Hence,

$$
\begin{aligned}
\|\mathrm{R}(\lambda, \mathcal{B}) z\|^{2} & \leq \sum_{n=1}^{\infty}\left\|\mathrm{K}_{n} \mathrm{P}_{n}\left(\lambda-\mathrm{J}_{n} \mathrm{P}_{n}\right)^{-1} \mathrm{~K}_{n}^{-1} \mathrm{P}_{\mathrm{n}}\right\|_{\mathrm{B}(\mathbb{X})}^{2}\left\|\mathrm{P}_{n} z\right\|^{2} \\
& \leq \sum_{n=1}^{\infty}\left\|\mathrm{K}_{n} \mathrm{P}_{n}\right\|_{\mathrm{B}(\mathbb{X})}^{2}\left\|\left(\lambda-\mathrm{J}_{n} \mathrm{P}_{n}\right)^{-1}\right\|_{\mathrm{B}(\mathbb{X})}^{2}\left\|\mathrm{~K}_{n}^{-1} \mathrm{P}_{n}\right\|_{\mathrm{B}(\mathbb{X})}^{2}\left\|\mathrm{P}_{n} z\right\|^{2} .
\end{aligned}
$$

$$
\begin{aligned}
& \text { Moreover, for } z:=\left(\begin{array}{c}
z_{1} \\
z_{2}
\end{array}\right) \in \mathbb{X} \text {, we obtain } \\
& \qquad \begin{aligned}
\left\|\mathrm{K}_{\mathrm{n}} \mathrm{P}_{\mathrm{n}} z\right\|^{2} & =\left\|\mathrm{E}_{\mathrm{n}} z_{1}+\mathrm{E}_{\mathrm{n}} z_{2}\right\|^{2}+\left\|\lambda_{1}^{n} \mathrm{E}_{\mathrm{n}} z_{1}+\lambda_{2}^{n} \mathrm{E}_{\mathrm{n}} z_{2}\right\|^{2} \\
& \leq 3\left(1+\left|\lambda_{1}^{n}\right|^{2}\right)\|z\|^{2} .
\end{aligned}
\end{aligned}
$$

Thus, there exists $C_{1}>0$ such that

$$
\left\|K_{n} P_{n} z\right\| \leq C_{1}\left|\lambda_{1}^{n}\right|\|z\| \text { for all } n \geq 1
$$

Similarly, for $z:=\left(\begin{array}{c}z_{1} \\ z_{2}\end{array}\right) \in \mathbb{X}$, one can show that there is $C_{2}>0$ such that

$$
\left\|K_{n}^{-1} P_{n} z\right\| \leq \frac{C_{2}}{\left|\lambda_{1}^{n}\right|}\|z\| \text { for all } n \geq 1
$$

Now, for $z \in \mathbb{X}$, we have

$$
\begin{aligned}
\left\|\left(\lambda-\mathrm{J}_{\mathrm{n}} \mathrm{P}_{\mathrm{n}}\right)^{-1} z\right\|^{2} & =\left\|\left(\begin{array}{cc}
\frac{1}{\lambda-\lambda_{1}^{n}} & 0 \\
0 & \frac{1}{\lambda-\lambda_{2}^{n}}
\end{array}\right)\left(\begin{array}{c}
z_{1} \\
z_{2}
\end{array}\right)\right\|^{2} \\
& \leq \frac{1}{\left|\lambda-\lambda_{1}^{n}\right|^{2}}\left\|z_{1}\right\|^{2}+\frac{1}{\left|\lambda-\lambda_{2}^{n}\right|^{2}}\left\|z_{2}\right\|^{2} .
\end{aligned}
$$

Let $\lambda_{0}>0$. Define the function

$$
\eta(\lambda):=\frac{1+|\lambda|}{\left|\lambda-\lambda_{2}^{n}\right|}
$$

It is clear that $\eta$ is continuous and bounded on the closed set

$$
\Sigma:=\left\{\lambda \in \mathbb{C}:|\lambda| \leq \lambda_{0},|\arg \lambda| \leq \omega\right\}
$$


On the other hand, it is clear that $\eta$ is bounded for $|\lambda|>\lambda_{0}$. Thus $\eta$ is bounded on $S_{\omega}$. If we take

$$
N=\sup \left\{\frac{1+|\lambda|}{\left|\lambda-\lambda_{\mathfrak{n}}^{j}\right|}: \lambda \in S_{\omega}, n \geq 1 ; j=1,2,\right\} .
$$

Therefore,

$$
\left\|\left(\lambda-\mathrm{J}_{\mathrm{n}} \mathrm{P}_{\mathrm{n}}\right)^{-1} z\right\| \leq \frac{\mathrm{N}}{1+|\lambda|}\|z\|, \quad \lambda \in \mathrm{S}_{\omega} .
$$

Consequently,

$$
\|\mathrm{R}(\lambda, \mathcal{B})\| \leq \frac{\mathrm{K}}{1+|\lambda|}
$$

for all $\lambda \in S_{\omega}$ and $t \in \mathbb{R}$.

In view of the above, $\mathcal{B}$ is sectorial. Let $\left(e^{\tau \mathcal{B}}\right)_{\tau \geq 0}$ be the nalytic semigroup associated with it. Let us show that $\left(e^{\tau \mathcal{B}}\right)_{\tau \geq 0}$ is exponentially stable.

Now

$$
e^{\tau \mathcal{B}} z=\sum_{n=0}^{\infty} K_{n}^{-1} P_{n} e^{\tau J_{n}} P_{n} K_{n} P_{n} z, z \in \mathbb{X} .
$$

On the other hand, we have

$$
\left\|e^{\tau \mathcal{B}} z\right\|=\sum_{n=0}^{\infty}\left\|K_{n}^{-1} P_{n}\right\|_{B(\mathbb{X})}\left\|e^{\tau J_{n}} P_{n}\right\|_{B(\mathbb{X})}\left\|K_{n} P_{n}\right\|_{B(\mathbb{X})}\left\|P_{n} z\right\|,
$$

with for each $z=\left(\begin{array}{l}z_{1} \\ z_{2}\end{array}\right)$

$$
\begin{aligned}
\left\|e^{\tau J_{n}} P_{n} z\right\|^{2} & =\left\|\left(\begin{array}{cc}
e^{\lambda_{1}^{n} \tau} E_{n} & 0 \\
& \\
0 & e^{\lambda_{2}^{n} \tau} E_{n}
\end{array}\right)\left(\begin{array}{c}
z_{1} \\
z_{2}
\end{array}\right)\right\|^{2} \\
& \leq\left\|e^{\lambda_{1}^{n} \tau} E_{n} z_{1}\right\|^{2}+\left\|e^{\lambda_{2}^{n} \tau} E_{n} z_{2}\right\|^{2} \\
& \leq e^{-a \tau}\|z\|^{2} .
\end{aligned}
$$

Therefore,

$$
\left\|e^{\tau \mathcal{B}}\right\| \leq \mathrm{C} e^{-\mathrm{a} \tau}, \quad \tau \geq 0 .
$$

It is now clear that if $L$ is small enough, then the second-order differential equation Eq. (1.3) has at most one solution

$$
\left(\begin{array}{l}
u \\
v
\end{array}\right) \in \mathbb{X}_{\alpha}=\mathbb{H}_{\alpha} \times \mathbb{H}
$$

which in addition is pseudo almost automorphic. Therefore, Eq. (1.2) has a unique solution $u \in \mathbb{H}_{\alpha}$, which in addition is pseudo almost automorphic. 


\section{Examples}

\subsection{1-Dimensional Sine-Gordon Equation}

Let $\mathrm{L}>0$ and and let $\mathrm{J}=(0, \mathrm{~L})$. Let $\mathbb{H}=\mathrm{L}^{2}(\mathrm{~J})$ be equipped with its natural topology. Our main objective here is to study the existence of pseudo almost automorphic solutions to a somewhat general one-dimensional Sine-Gordon equation with Dirichlet boundary conditions, which had been studied in the literature especially by Leiva 36 in the following form

$$
\begin{gathered}
\frac{\partial^{2} u}{\partial t^{2}}+c \frac{\partial u}{\partial t}-d \frac{\partial^{2} u}{\partial x^{2}}+k \sin u=p(t, x), \quad t \in \mathbb{R}, \quad x \in J \\
u(t, 0)=u(t, L)=0, \quad t \in \mathbb{R}
\end{gathered}
$$

where $\mathrm{c}, \mathrm{d}, \mathrm{k}$ are positive constants, $\mathrm{p}: \mathbb{R} \times \mathrm{J} \mapsto \mathbb{R}$ is continuous and bounded.

Precisely, we are interested in the following system of second-order partial differential equations

$$
\begin{gathered}
\frac{\partial^{2} u}{\partial t^{2}}+a \frac{\partial u}{\partial t}-b \frac{\partial^{2} u}{\partial x^{2}}=Q(t, x, u), \quad t \in \mathbb{R}, \quad x \in J \\
u(t, 0)=u(t, L)=0, \quad t \in \mathbb{R}
\end{gathered}
$$

where $\mathrm{a}, \mathrm{b}>0$ and $\mathrm{Q}: \mathbb{R} \times \mathrm{J} \times \mathrm{L}^{2}(\mathrm{~J}) \mapsto \mathrm{L}^{2}(\mathrm{~J})$ is pseudo-almost automorphic.

Let us take

$$
A v=-v^{\prime \prime} \text { for all } u \in D(A)=\mathbb{H}_{0}^{1}(J) \cap \mathbb{H}^{2}(J)
$$

and suppose that $\mathrm{Q}: \mathbb{R} \times \mathrm{J} \times \mathrm{L}^{2}(\mathrm{~J}) \mapsto \mathbb{H}_{0}^{\beta}(\mathrm{J})$ is pseudo-almost automorphic. Moreover, $\mathrm{Q}$ is Lipschitz in the following sense: there exists $L^{\prime \prime}>0$ for which

$$
\|\mathrm{Q}(\mathrm{t}, x, \mathrm{u})-\mathrm{Q}(\mathrm{t}, x, v)\|_{\mathbb{H}_{0}^{\beta}(\mathrm{J})} \leq \mathrm{L}^{\prime \prime}\|\mathrm{u}-v\|_{2}
$$

for all $u, v \in \mathrm{L}^{2}(J), x \in J$ and $t \in \mathbb{R}$.

Consequently, the system Eq. (6.3) - Eq. (6.4) has at most one solution $u \in \operatorname{PAA}\left(\mathbb{H}_{0}^{1}(J)\right)$ when $L^{\prime \prime}$ is small enough.

\subsection{N-dimensional Sine-Gordon Equation}

Let $\Omega \subset \mathbb{R}^{N}(N \geq 1)$ be a open bounded subset with $C^{2}$ boundary $\Gamma=\partial \Omega$ and let $\mathbb{H}=\mathrm{L}^{2}(\Omega)$ equipped with its natural topology $\|\cdot\|_{\mathrm{L}^{2}(\Omega)}$. Here, we are interested in the $\mathrm{N}$-dimensional SineGordon studied in the previous example, that is, the system of second-order partial differential equations given by 


$$
\begin{gathered}
\frac{\partial^{2} u}{\partial t^{2}}+a \frac{\partial u}{\partial t}-b \Delta u=R(t, x, u), \quad t \in \mathbb{R}, \quad x \in \Omega \\
u(t, x)=0, \quad t \in \mathbb{R}, \quad x \in \partial \Omega
\end{gathered}
$$

where $a, b>0$, and $R: \mathbb{R} \times \Omega \times \mathrm{L}^{2}(\Omega) \mapsto \mathrm{L}^{2}(\Omega)$ is jointly continuous.

Define the linear operator $A$ as follows:

$$
A u=-\Delta u \text { for all } u \in D(A)=\mathbb{H}_{0}^{1}(\Omega) \cap \mathbb{H}^{2}(\Omega) .
$$

For each $\mu \in(0,1)$, we take $\mathbb{H}_{\mu}=\mathrm{D}\left((-\Delta)^{\mu}\right)=\mathbb{H}_{0}^{\mu}(\Omega) \cap \mathbb{H}^{2 \mu}(\Omega)$ equipped with its $\mu$-norm $\|\cdot\|_{\mu}$.

Suppose that $R: \mathbb{R} \times \Omega \times \mathrm{L}^{2}(\Omega) \mapsto \mathbb{H}_{0}^{\beta}(\Omega)$ is pseudo-almost automorphic. Moreover, $\mathrm{R}$ is Lipschitz in the following sense: there exists $L^{\prime \prime \prime}>0$ for which

$$
\|R(t, x, u)-R(t, x, v)\|_{\beta} \leq L^{\prime \prime \prime}\|u-v\|_{2}
$$

for all $u, v \in \mathrm{L}^{2}(\Omega), x \in \Omega$ and $\mathrm{t} \in \mathbb{R}$.

Therefore, the system Eq. (6.5) - Eq. (6.6) has at most one solution $u \in P A A\left(\mathbb{H}_{0}^{1}(\Omega)\right)$ when $\mathrm{L}^{\prime \prime \prime}$ is small enough.

Received: July 2010. Revised: August 2010.

\section{References}

[1] P. Acquistapace, Evolution operators and strong solutions of abstract linear parabolic equations. Differential Integral Equations 1 (1988), pp. 433-457.

[2] P. Acquistapace, F. Flandoli, B. Terreni, Initial boundary value problems and optimal control for nonautonomous parabolic systems. SIAM J. Control Optim. 29 (1991), pp. 89-118.

[3] P. Acquistapace, B. Terreni, A unified approach to abstract linear nonautonomous parabolic equations. Rend. Sem. Mat. Univ. Padova 78 (1987), pp. 47-107.

[4] J. M. Alonso and R. Ortega, Global Aymptotic Stability of a Forced Newtonian System with Dissipation. J. Math. Anal. Appl. (1995), pp. 965-986.

[5] J. M. Alonso, J. Mawhin, and R. Ortega, Bounded Solutions of Second-Order Semilinear Evolution Equations and Applications to the Telegraph Equation. J. Math. Pures Appl. (1999), pp. $43-63$.

[6] H. Amann, Linear and Quasilinear Parabolic Problems, Birkhäuser, Berlin 1995. 
[7] B. Amir and L. Maniar, Existence and some asymptotic behaviors of solutions to semilinear Cauchy problems with non dense domain via extrapolation spaces, Rend. Circ. Mat. Palermo (2000), pp. 481-496.

[8] B. Amir and L. Maniar, Composition of Pseudo-Almost Periodic Functions and Cauchy Problems with Perator of Nondense Domain. Ann. Math. Blaise Pascal 6 (1999), no. 1, pp. 1-11.

[9] W. Arendt, R. Chill, S. Fornaro, and C. Poupaud, Lp-Maximal Regularity for NonAutonomous Evolution Equations. J. Differential Equations 237 (2007), no. 1, pp. 1-26.

[10] W. Arendt and C. J. K. Batty, Almost Periodic Solutions of First- and Second-Order Cauchy Problems. J. Differential Equations 137 (1997), no. 2, pp. 363-383.

[11] J. Blot, P. Cieutat, and J. Mawhin, Almost Periodic Oscillation of Monotone Second-Order Systems. Advances Diff. Equ. 2 (1997), no. 5, pp. 693-714.

[12] M. Baroun, S. Boulite, T. Diagana, and L. Maniar, Almost periodic solutions to some semilinear non-autonomous thermoelastic plate equations. J. Math. Anal. Appl. 349(2009), no. 1, pp. $74-84$.

[13] M. Baroun, S. Boulite, G. M. N'Guérékata, and L. Maniar, Almost automorphy of Semilinear Parabolic Equations. Electron. J. Differential Equations 2008(2008), no. 60, pp. 1-9.

[14] S. Boulite, L. Maniar, and G. M. N'Guérekata, Almost Automorphic Solutions for Hyperbolic Semilniear Evolution Equations, Semigroup Forum. Vol. 71 (2005), pp. 231-240.

[15] D. Bugajewski and T. Diagana, Almost Automorphy of the Convolution Operator and Applications to Differential and Functional-Differential Equations, Nonlinear Stud. 13 (2006), no. 2, pp. 129-140.

[16] D. Bugajewski, T. Diagana, C. M. Mahop, Asymptotic and Pseudo Almost Periodicity of the Convolution Operator and Applications to Differential and Integral Equations. Z. Anal. Anwend. 25 (2006), no. 3, pp. 327-340.

[17] C. Chicone, Y. Latushkin, Evolution Semigroups in Dynamical Systems and Differential Equations. Amer. Math. Soc., 1999.

[18] P. Cieutat and K. Ezzinbi, Existence, uniqueness and attractiveness of a pseudo almost automorphic solutions for some dissipative differential equations in Banach spaces. J. Math. Anal. Appl. 354 (2009), no. 2, pp. 494-506.

[19] G. Da Prato and P. Grisvard, Equations d'évolution abstraites non linéaires de type parabolique. Ann. Mat. Pura Appl. (4) 120 (1979), pp. 329-396.

[20] T. Diagana, Existence of pseudo-almost automorphic solutions to some abstract differential equations with $S^{\mathfrak{p}}$-pseudo-almost automorphic coefficients. Nonlinear Anal. $\mathbf{7 0}$ (2009), no. 11, pp. 3781-3790. 
[21] T. Diagana, Pseudo almost periodic functions in Banach spaces. Nova Science Publishers, Inc., New York, 2007.

[22] T. Diagana and E. Hernàndez M., Existence and Uniqueness of Pseudo Almost Periodic Solutions to Some Abstract Partial Neutral Functional-Differential Equations and Applications, J. Math. Anal. Appl. 327(2007), no. 2, pp. 776-791.

[23] T. Diagana, Existence of pseudo almost periodic solutions to some classes of partial hyperbolic evolution equations. Electron. J. Qual. Theory Differ. Equ. 2007, No. 3, 12 pp.

[24] T. Diagana, Existence and Uniqueness of Pseudo Almost Periodic Solutions to Some Classes of Partial Evolution Equations. Nonlinear Anal. 66 (2007), no. 2, pp. 384-395.

[25] T. Diagana and G. M. N'Guérékata, Pseudo Almost Periodic Mild Solutions To Hyperbolic Evolution Equationa in Abstract Intermediate Banach Spaces. Applicable Anal. 85 (2006), Nos. 6-7, pp. 769-780.

[26] T. Diagana, N. Henríquez, and E. Hernàndez, Almost automorphic mild solutions to some partial neutral functional-differential Equations and Applications. Nonlinear Anal. 69 (2008), no. 5, pp. 1485-1493.

[27] T. Diagana and G. M. N'Guérékata, Almost automorphic solutions to some classes of partial evolution equations. Appl. Math. Lett. 20 (2007), no. 4, pp. 462-466.

[28] K. J. Engel and R. Nagel, One Parameter Semigroups for Linear Evolution Equations, Graduate texts in Mathematics, Springer Verlag 1999.

[29] K. Ezzinbi, S. Fatajou and G. M. NGuérékata, Pseudo almost automorphic solutions to some neutral partial functional differential equations in Banach space. Nonlinear Anal. 70 (2009), no. 4, pp. 1641-1647.

[30] K. Ezzinbi, S. Fatajou and G. M. NGuérékata, Pseudo almost automorphic solutions for dissipative differential equations in Banach spaces. J. Math. Anal. Appl. 351 (2009), no. 2, pp. 765-772.

[31] A. M. Fink, Almost Periodic Differential Equations, Lecture Notes in Mathematics 377, Springer-Verlag, New York-Berlin, 1974.

[32] E. Hernández and H. R. Henríquez, Existence of Periodic Solutions of Partial neutral Functional Differential Equations with Unbounded Delay. J. Math. Anal. Appl 221 (1998), no. 2, pp. 499-522.

[33] E. Hernández, Existence Results for Partial Neutral Integro-differential Equations with Unbounded Delay. J. Math. Anal. Appl 292 (2004), no. 1, pp. 194-210. 
[34] E. Hernández M., M. L. Pelicer, and J. P. C. dos Santos , Asymptotically Almost Periodic and Almost Periodic Solutions for a Class of Evolution Equations, Electron. J. Diff. Eqns 2004(2004), no. 61, pp. 1-15.

[35] Y. Hino, T. Naito, N. V. Minh, and J. S. Shin, Almost Periodic Solutions of Differential Equations in Banach Spaces. Stability and Control: Theory, Methods and Applications, 15. Taylor and Francis, London, 2002.

[36] H. Leiva, Existence of Bounded Solutions Solutions of a Second-Order System with Dissipation. J. Math. Anal. Appl. 237 (1999), pp. 288-302.

[37] H. Leiva and Z. Sivoli, Existence, Stability and Smoothness of a Bounded Solution for Nonlinear Time-Varying Theormoelastic Plate Equations. J. Math. Anal. Appl. 285 (1999), pp. 191-211.

[38] J.-L. Lions and J. Peetre, Sur une classe d'espaces d'interpolation. Inst. Hautes tudes Sci. Publ. Math. no. 19 (1964), pp. 5-68.

[39] J. Liang, J. Zhang, and T-J. Xiao, Composition of Pseudo Almost Automorphic and Asymptotically almost automorphic functions. J. Math. Anal. Appl. 340 (2008), pp. 1493-1499.

[40] J. Liang, G. M. N'Guérékata, T-J. Xiao, and J. Zhang, Some properties of pseudo almost automorphic functions and applications to abstract differential equations. Nonlinear Anal. 70 (2009), no. 7, pp. 2731-2735.

[41] A. Lunardi, Analytic Semigroups and Optimal Regularity in Parabolic Problems, PNLDE Vol. 16, Birkhäauser Verlag, Basel, 1995.

[42] L. Maniar, R. Schnaubelt, Almost periodicity of inhomogeneous parabolic evolution equations, Lecture Notes in Pure and Appl. Math. vol. 234, Dekker, New York (2003), 299-318.

[43] J. Mawhin, Bounded Solutions of Second-Order Semicoercive Evolution Equations in a Hilbert Space and Nonlinear Telegraph Equations. Rend. Sem. Mat. Univ. Pol. Torino. 58 (2000), no. 3, pp. 361-374.

[44] M. G. Naso, A. Benabdallah, Thermoelastic plate with thermal interior control, Mathematical models and methods for smart materials (Cortona, 2001), 247-250, Ser. Adv. Math. Appl. Sci., 62, World Sci. Publ., River Edge, NJ, 2002.

[45] A. W. Naylor and G. R. Sell, Linear Operator Theory in Engineering and Science. Applied Mathematical Sciences 40, Springer-Verlag, 1971.

[46] G. M. N'Guérékata, Almost automorphic functions and almost periodic functions in abstract spaces, Kluwer Academic / Plenum Publishers, New York-London-Moscow, 2001.

[47] A. Pazy, Semigroups of linear operators and applications to partial differential equations, Applied Mathematical Sciences, 44. Springer-Verlag, New York, 1983. 
[48] J. Prüss, Evolutionary Integral Equations and Applications, Birkhäuser, 1993.

[49] R. Schnaubelt, Sufficient conditions for exponential stability and dichotomy of evolution equations. Forum Math. 11(1999), pp. 543-566.

[50] R. Schnaubelt, Asymptotically autonomous parabolic evolution equations, J. Evol. Equ. 1 (2001), pp. 19-37.

[51] R. Schnaubelt, Asymptotic behavior of parabolic nonautonomous evolution equations, in: M. Iannelli, R. Nagel, S. Piazzera (Eds.), Functional Analytic Methods for Evolution Equations, in: Lecture Notes in Math., 1855, Springer-Verlag, Berlin, 2004, pp. 401-472.

[52] T. J. Xiao, J. Liang, J. Zhang, Pseudo almost automorphic solutions to semilinear differential equations in Banach spaces. Semigroup Forum 76 (2008), no. 3, pp. 518-524.

[53] T. J. Xiao, X-X. Zhu, J. Liang, Pseudo-almost automorphic mild solutions to nonautonomous differential equations and applications. Nonlinear Anal. 70 (2009), no. 11, pp. 4079-4085.

[54] T. J. Xiao, J. Liang, Second-order linear differential equations with almost periodic solutions, Acta Math. Sinica (N.S.) 7 (1991), 354-359.

[55] T. J. Xiao, J. Liang, Complete second-order linear differential equations with almost periodic solutions, J. Math. Anal. Appl. 163 (1992), 136-146.

[56] T. J. Xiao, J. Liang, The Cauchy Problem for Higher-Order Abstract Differential Equations, Lecture Notes in Mathematics, Vol. 1701, Springer, Berlin, 1998.

[57] A. Yagi, Parabolic equations in which the coefficients are generators of infinitely differentiable semigroups II, Funkcial. Ekvac. 33 (1990), pp. 139-150.

[58] A. Yagi, Abstract quasilinear evolution equations of parabolic type in Banach spaces, Boll. Un. Mat. Ital. B (7) 5 (1991), pp. 341-368.

[59] S. Zaidman, Topics in Abstract Differential Equations, Pitman Research Notes in Mathematics Ser. II John Wiley and Sons, New York, 1994-1995. 